researchers also measured the mass of bacteria and synthetic micro- and nanoparticles in transit through the suspended microchannels by matching pressures at the inlet and the outlet of the resonator, which reduced the flow rate and increased the transit time of the particle through the device, enabling higher resolution measurements of frequency shifts. This mode required no chemical modification of the resonator and was not affected by sensor drift or bulk density changes, allowing the researchers to run a large number of assays without surface regeneration.

The suspended microchannel resonator enables the analysis of precious samples with minimal reagent consumption; can implement differential sensing and temperature control to improve the detection of small signals in the presence of thermal drift, non-specific binding, or bulk density differences; and can be integrated in elastomeric microfluidic systems, which can provide pumps and valves to deliver fluids to the detector with great precision, the researchers said. They envision that flowthrough resonant mass sensing could be configured for counting specific cells in a similar way as flow cytometry or for determining the size and mass density of colloidal particles. Mass labelling would enable the specific detection of pathogens such as bacteria, viruses, or toxins with appropriate functionalization of the surfaces of the microchannels.

JOAN J. CARVAJAL

\section{Spontaneous Folding of PDMS Membranes Achieved}

Microfabrication can be a difficult and expensive proposition; methods that take advantage of spontaneous, self-assembled processes can potentially simplify microfabrication. C. Py and a team of researchers from The City of Paris Industrial Physics and Chemistry Higher Educational Institution (ESPCI) and the Hydrodynamics Laboratory (LadHyX) of the Ecole Polytechnique in Palaiseau, France have reported in the April 13 issue of Physical Review Letters (DOI:10.1103/PhysRevLett.98. 156103) a method to shape sub-millimeter objects by wrapping thin films around water droplets. Water droplets deposited on a small, shaped polymeric film induced capillary forces that were strong enough to counteract the elastic energy of the film and distort the shape. The researchers explored the phenomenon experimentally and offer a model for predicting the critical length scales based on an energy balance between the interfacial and elastic energy of the film.

In order to investigate the effects of capillary forces on elastic thin films, the researchers spin-coated polydimethyl- siloxane (PDMS) films on glass slides. These thin films (40-80 $\mu \mathrm{m}$ in thickness) were then cut and a droplet of water was deposited. Figure 1 shows films cut into square, triangle, and cross shapes leading to spontaneously formed tubular, pyramidal, and cubic volumes, respectively, as the water droplet evaporates. For films of appropriate dimensions, the film encapsulates the water droplet and then, as the droplet dries, the polymer assumes a three-dimensional shape determined by the two-dimensional cutout. By numerically evaluating the differential elastic and interfacial equations and matching them to experimental data, an elasto-capillary length was determined, $L_{\mathrm{EC}}=$ $\left(E h^{3} /\left[12\left(1-v^{2}\right) \gamma\right]\right)^{1 / 2}$, where $E$ is Young's modulus, $h$ is the film thickness, $v$ is Poisson's ratio and $\gamma$ is the surface ten- sion of the liquid. Above this critical length, a shape is successfully folded. Py notes that the $h^{3 / 2}$ dependence of $L_{\mathrm{EC}}$ is beneficial to miniaturization as the critical length scale becomes even smaller as the thickness decreases.

Efforts to create more complex shapes will require more sophisticated models to account for gravitational forces and threedimensional stresses. The researchers acknowledge the inevitable problem of crumpling, stemming from geometric incompatibilities between planes and volumes described in Gauss's theorema egregium, but point out the possible ways this may be overcome. The ability to create complex shapes from planar cutouts will make micro- and nano-fabrication cheaper, easier, and more accessible.

ARTHUR FELDMAN
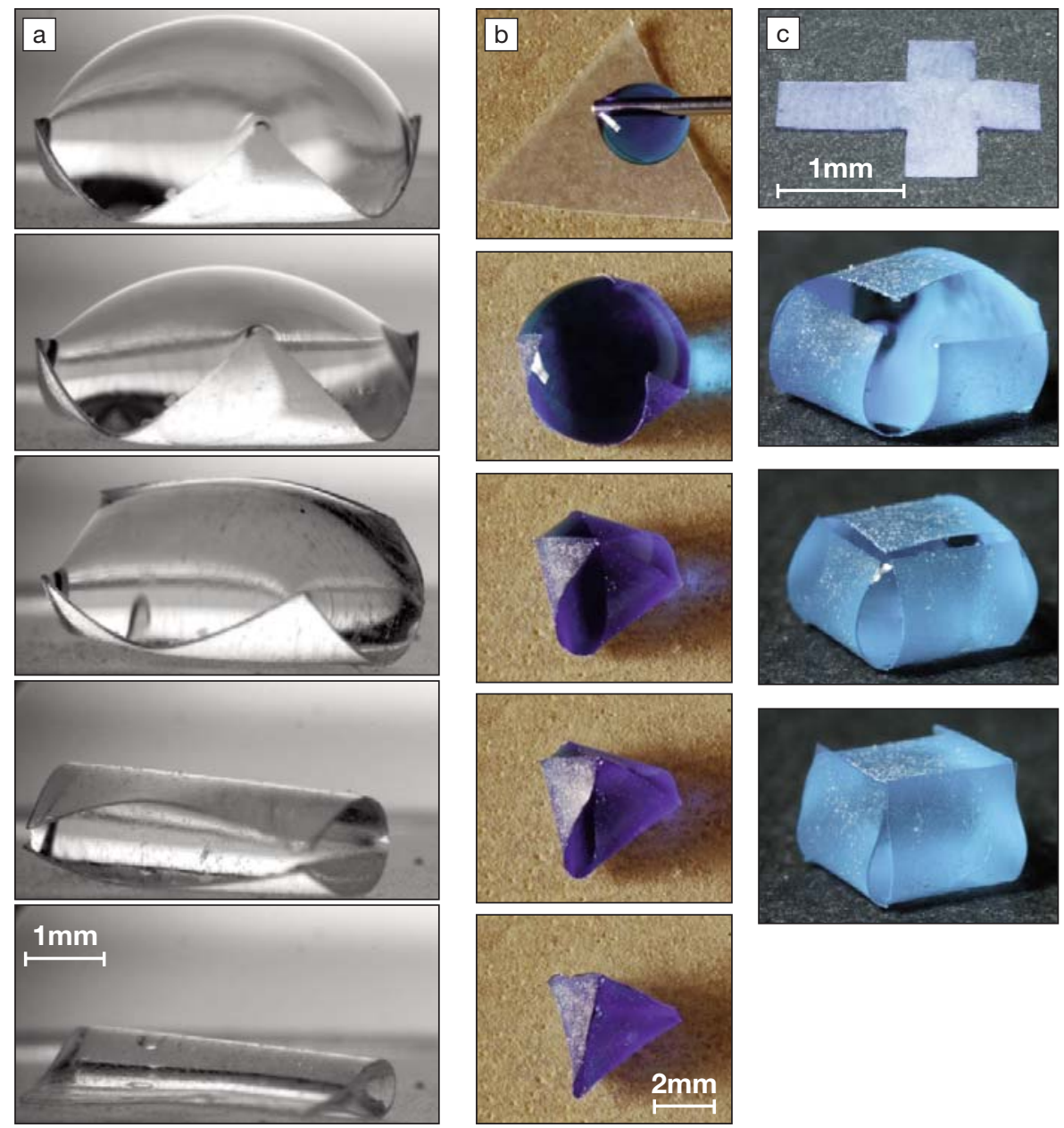

Figure 1. Two-dimensional cutouts of polydimethylsiloxane (PDMS) films assume threedimensional shapes: (a) square to tube, (b) triangle to pyramid, and (c) cross to cube. Videos of these formations are available at www.aip.org/pubservs/epaps.html. Reprinted with permission from C. Py, P. Reverdy, L. Doppler, J. Bico, B. Roman, and C.N. Baroud, Phys. Rev. Lett. 98, 156103 (2007). (C2007 American Physical Society. 